\title{
Geodesic Distance on Riemannian Manifold using Jacobi Iterations in 3D Face Recognition system
}

\author{
Rachid Ahdid $^{1 *}$, Said Safi ${ }^{1}$, Mohamed Fakir ${ }^{2,}$ Bouzid Manaut $^{3}$ \\ ${ }^{1}$ Department of Mathematics and Informatics, Polydisciplinary Faculty, Sultan Moulay Slimane University, Morocco \\ ${ }^{2}$ Departement Informatics, Faculty of Science and Technology, Sultan Moulay Slimane University, Morocco. \\ ${ }^{3}$ Departement of Physics, Polydisciplinary Faculty, Sultan Moulay Slimane University, Morocco.
}

\begin{tabular}{l} 
Article Info \\
\hline Article history: \\
Received Jan 13, 2017 \\
Revised Feb 25, 2017 \\
Accepted Mar 17, 2017 \\
\hline Keywords: \\
3D face recognition \\
Eikonal equation \\
Geodesic distance \\
Jacobi iterations \\
Riemannian geometry \\
\hline
\end{tabular}

\begin{abstract}
In this paper, we present an automatic application of $3 \mathrm{D}$ face recognition system using geodesic distance in Riemannian geometry. We consider, in this approach, the three dimensional face images as residing in Riemannian manifold and we compute the geodesic distance using the Jacobi iterations as a solution of the Eikonal equation. The problem of solving the Eikonal equation, unstructured simplified meshes of 3D face surface, such as tetrahedral and triangles are important for accurately modeling material interfaces and curved domains, which are approximations to curved surfaces in $\mathrm{R}^{3}$. In the classifying steps, we use: Neural Networks (NN), K-Nearest Neighbor (KNN) and Support Vector Machines (SVM). To test this method and evaluate its performance, a simulation series of experiments were performed on 3D Shape REtrieval Contest 2008 database (SHREC2008).
\end{abstract}

Copyright $@ 2017$ Institute of Advanced Engineering and Science. All rights reserved.

\section{Corresponding Author:}

Rachid Ahdid,

Departement of Mathematics and Informatics,

Sultan Moulay Slimane University,

Polydisciplinary Faculty, Sultan Moulay Slimane University, Beni Mellal, Morocco.

Email: r.ahdid@usms.ma

\section{INTRODUCTION}

Face recognition is one of the most commonly used techniques in biometrics authentication applications, of access and video surveillance control, this is due to its advantageous features. Face recognition is one of the few biometric methods that possess the merits of both high accuracy and low intrusiveness. It has the accuracy of a physiological approach without being intrusive. For this reason, face recognition has drawn the attention of researchers in field from image processing to computer vision.

In these last years, a number of methods have been proposed for the recognition of human faces. In spite of the results obtained in this domain, the automatic face recognition stays one of very difficult problem. Several methods were developed for 2D face recognition. However, it has a certain number of limitations related to the orientation of the face or laying, lighting, facial expression. These last years, we talk more and more about 3D face recognition technology as solutions of 2D face recognition problems.

Many approaches to 3D face recognition have been proposed during the past thirty years. Chua et al. [1] use "point signatures" in 3D face recognition. Point signatures are used to locate reference points that are used to standardize the pose. Achermann and Bunke [2] report on a method of 3D face recognition that uses an extension of Hausdorff distance matching. Moreno and co-workers [3] approach 3D face recognition by first performing a segmentation based on Gaussian curvature and then creating a feature vector based on the segmented regions. Lee et al. [4] perform 3D face recognition by locating the nose tip, and then forming a feature vector based on contours along the face at a sequence of depth values. Medioni and Waupotitsch [5] perform 3D face recognition using an iterative closest point (ICP) approach to match face surfaces. Whereas most of the works covered here use 3D shapes acquired through a structured-light sensor, this work uses 3D 
shapes acquired by a passive stereo sensor. Xu et al. [6] developed a method for 3D face recognition using the original 3D point cloud converted to a regular mesh. The nose region is found and used as an anchor to find other local regions. A feature vector is computed from the data in the local regions of mouth, nose, left eye, and right eye. Feature space dimensionality is reduced using principal components analysis, and matching is based on minimum distance using both global and local shape components. Lu et al. [7] report on results of an ICP-based approach to 3D face recognition. This approach assumes that the gallery 3D image is a more complete face model and the probe 3D image is a frontal view that is likely a subset of the gallery image. Pan et al. [8] apply PCA, or eigenface, matching to a novel mapping of the 3D data to a range, or depth, image. Finding the nose tip to use as a center point, and an axis of symmetry to use for alignment, the face data are mapped to a circular range image. Lee et al. [9] propose an approach to 3D face recognition based on the curvature values at eight feature points on the face. Using a Support Vector Machine for classification. They use a Cyberware sensor to acquire the enrollment images and a Genex sensor to acquire the probe images. Y. Wang et al. [10] used Iterative Closest Point (ICP) algorithm. In 3D face recognition, ICP is frequently used for surface registration. Samir et al. [11] represented facial surfaces using a union of level-set curves of the depth function with respect to the nose tip named isodepth curves and constructed a shape space of curves of interest. Feng et al. [12] divided the isogeodesics in small segments of equal arc length that form the basis of trained face signatures. So, they focused on local regions to make the signature independent of the starting point of a curve. They used the Fels-Olver construction and the 3D analog of Hann-Hickerman integral variables to derive integral invariants for curves in 3D subjected to the Euclidean group. Jahanbin et al. [13] extracted from each isogeodesic five shape descriptors: convexity, ratio of principal axes, compactness, circular and elliptic variance. These features are trained with Linear Discriminant Analysis (LDA) and Support Vector Machines (SVM). Han et al. [14] using five manually identified landmark points within a sub surface composing of eyes and whole nose. Drira et al. [15] projected past work in Riemannian analysis of shapes of closed curves on nasal surfaces. This choice is due to the stability of nose data collection and the invariance of nasal shape under expressions. Maalej et al. [16] presented another application for facial expression recognition, they extracted several relevant regions of a given facial surfaces. They locally projected isogeodesic path idea by representing each patch with a set of closed curves.

In this work we present an automatic 3D face recognition system based on facial surface analysis using a Riemannian geometry. For this we take the following steps: (1)- Nose tip detection as a reference point of 3D face. (2)- Geodetic distance Computing between the reference point and the other points of the 3D facial surface using the Jacobi iterations. (3)- Geodesic distances space matrices reduction using Principal Component Analysis (PCA) or Linear Discriminant Analysis (LDA) algorithms.

The rest of this paper is organized as follows: Section 2 describes the methodology of the proposed method with its stapes. Section 3 includes the simulation results and method analysis. Section 4 conclusion and future works.

\section{RESEARCH METHOD}

The objective of this work is to perform an automatic 3D face recognition system. For this, we characterize each face by a matrix, whose elements are a set of geometric features (geodesic distance computing between reference point and other points of face surface). These matrices are finally used to recognize 3D faces under the framework of sparse representation. Figure (1) illustrates the steps of our proposed method.

Our system is divided on three main steps: Preprocessing, in this first step, we discretize a 3D face surface using a triangular mesh by the idea of displaced subdivision surfaces proposed by Lee et al [21] and Xiaoxing Li et al [22], and we detect automatically the reference point (nose tip). In the second step (feature extraction), we compute the geodesic distance between the reference point and all other points of 3D face surface to represent the 3D face image with a matrix whose elements are geodesic distance values. The geodesic distance between two points on a mesh surface is computed as the length of the shortest path connecting the two points while remaining on the facial surface. We compute the geodesic distance between two points on a mesh surface using the Jacobi iterations as a solution of the Eikonal equation. Learning and Classification, in this step we use three types of classification algorithms such as: Neural Networks (NN), KNearest Neighbor (KNN) and Support Vector Machines (SVM). Figure (1) illustrates the steps of our proposed method. 


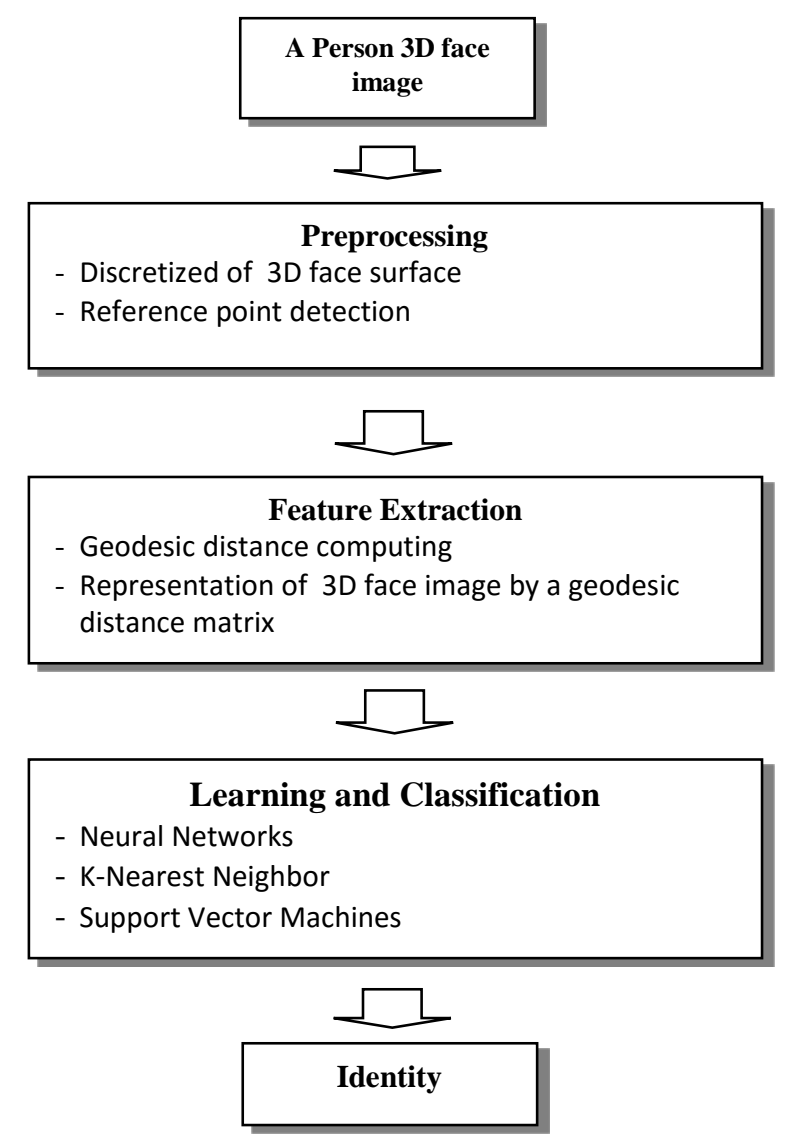

Figure 1. Methodology Architecture

\subsection{D Face Surface Subdivision}

Subdivision surface is curved surface described by a simple polygon control mesh and some subdivision rules. The idea of representing a surface as a displacement function was first introduced by Cook [18]. A subdivision surface is generated by iteratively refining a coarse control mesh driven by a refinement operator. In this work, we discretize a $3 \mathrm{D}$ face surface using a triangular mesh by the idea of displaced subdivision surfaces proposed by Lee et al [21] and Xiaoxing Li et al [22].

We consider a 3D face surface discretized using a triangular mesh with $\mathrm{N}$ vertices. Figure (3) shows a 3D face image of the Shape REtrieval Contest 2008 (SHREC2008) database whose obverse surface is discretized into a triangular mesh.

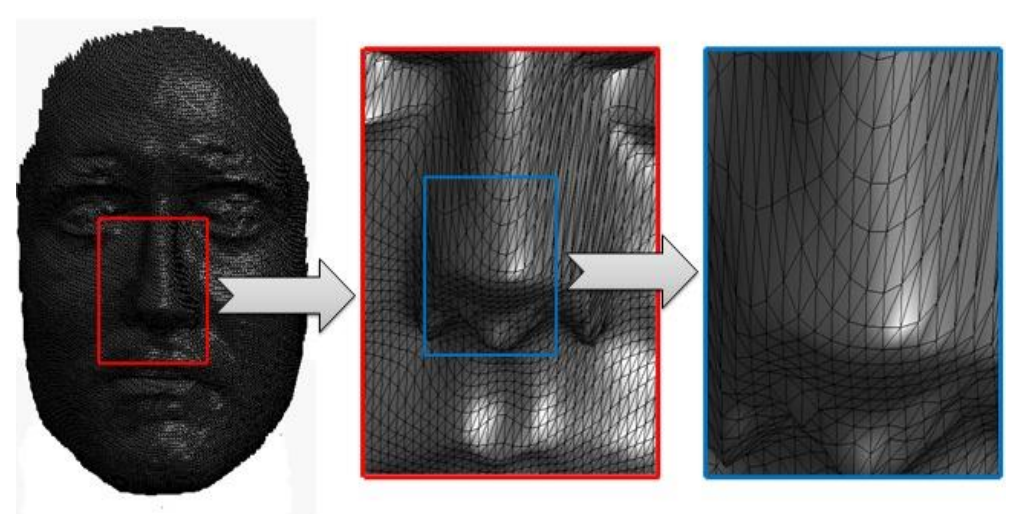

Figure 2. 3D facial surface discretized on triangular mesh of $\mathrm{N}$ vertices 


\subsection{Reference Point Detection}

The reference point (nose tip) is detected manually or automatically. There are several automatic approaches: L. Ballihi et al are developed an automatic algorithm nose end detection of a 3D face in [19]. This algorithm is based on two cuts of the facial surface. The first is at transverse face of the mass center. The second cut is based on the minimum depth point of the horizontal curve obtained by the first cut. The output of the last cut is a vertical curve and the minimum depth of this curve is the end of 3D face nose. In [20] S. Bahanbin et al use Gabor filters to automatically detect the nose tip. Another method has been used by C. Xu et al in 2004 [21], this method computes the effective energy of each neighbor pixel, then be determined the mean and variance of each neighbor pixel and uses the SVM to specify point end of the nose. L.H. Anuar et al [22] use a local geometry curvature and point signature to detect a nose tip region in 3D face model. In this work we have detected the point of reference $\mathrm{p}_{0}$ (nose tip) manually. Figure 1 summarizes the steps to detect the nose tip of a 3D face an image of the database SHREC2008:

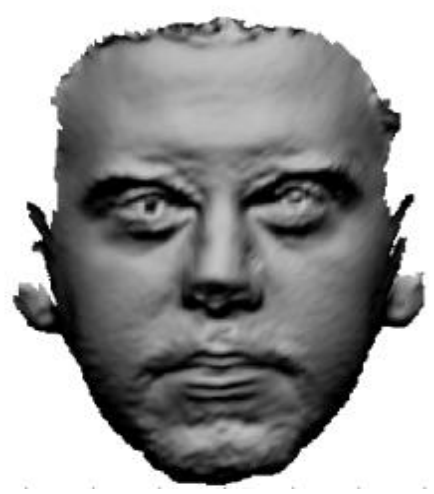

(a)

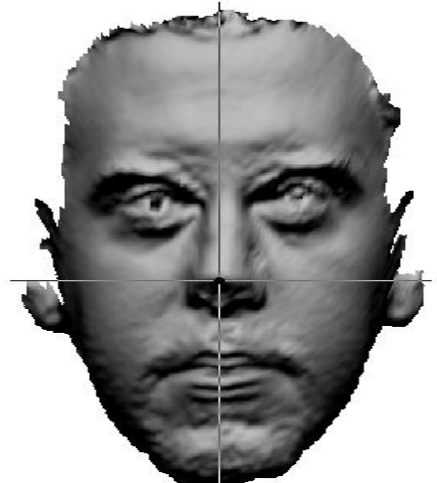

(b)

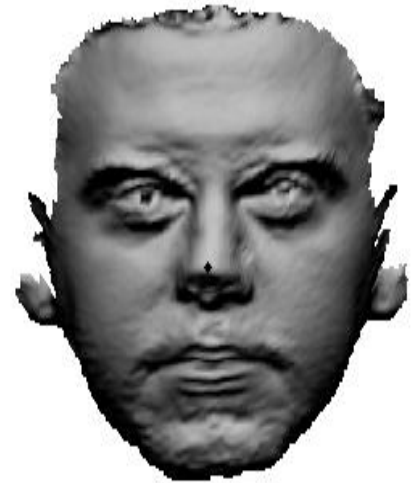

(c)

Figure 3. 3D face nose end detection steps: (a) 3D face image; (b) Manual nose tip selection; (c) Reference point detection

\subsection{Eikonal equation}

The Eikonal equation is of the form:

$$
\left|\nabla_{\mathrm{u}}(\mathrm{x})\right|=\mathrm{F}(\mathrm{x}) ; \mathrm{x} \in \Omega
$$

where:

$\Omega$ is an open set in Rn housebroken limit

$\nabla$ denote the gradient

|.| is the Euclidean norm

The Jacobi iteration method is a numerical method for solving boundary value problems of the Eikonal equation $[23,24,25]$. The algorithm is similar to the Dijkstra's algorithm and uses that information flows only to the outside from the planting area. We consider a 3D face surface discretized using a triangular mesh with N vertices. Figure (3) shows a 3D face image of the Shape REtrieval Contest 2008 (SHREC2008) database whose obverse surface is discretized into a triangular mesh.

\subsection{Eikonal Equation Discretization}

The discretized equation of the Eikonal equation is given with:

$$
\left\{\begin{array}{c}
\forall x \in \Omega, U(x)=\min _{y \in B(x)} U(y)+d(y, x), \\
\forall x \in S, U(x)=0,
\end{array}\right.
$$

where, $d(y, x)$ is the geodesic distance and $U(x)$ depends only on values of $U$ on neighbors y with $U(y)<$ $U(x)$. This will be a key obse vation in order to speed up the computation of $U$. 
To compute numerically a discrete geodesic distance map, we suppose that the manifold $\Omega$ is sampled using a set of points $\left\{\mathrm{x}_{\mathrm{i}}\right\}_{\mathrm{i}=0}^{\mathrm{N}-1} \subset \Omega$. The metric of the manifold is supposed to be sampled on this grid, and we denote the discrete metric.

$$
\mathrm{T}_{\mathrm{i}}=\mathrm{T}_{\mathrm{x}_{\mathrm{i}}} \in \mathrm{R}^{3 \times 3}
$$

We denote $\varepsilon$ the precision of the sampling. To derive a discrete counterpart to the Eiknal equation, each point $\mathrm{x}_{\mathrm{i}}$ is connected to its neighboring points $x_{\mathrm{j}} \in \operatorname{Neigh}\left(x_{i}\right)$. Each point is associated with a small surrounding neighborhood $B_{\varepsilon}\left(x_{i}\right)$, that is supposed to be a disjoint union of simplexes whose extremely vertices are the grid points $\left\{x_{i}\right\}_{i}$.

A distance map $U_{S(x)}$ for $x \in \Omega$ is approximated numerically by computing a discrete vector $u \in R^{N}$ where each value ui is intended to approximate the value of $U_{S(x i)}$.

This discrete vector is computed as a solution of a finite dimensional fixed point equation that discretizes (2). To that end, a continuous function $u(x)$ is obtained from the discrete samples $\left\{u_{i}\right\}_{i}$ by linear interpolation over the triangles.

We compute the minimization in (2) at the point $x=x_{i}$ over the boundary of $B_{\varepsilon}\left(x_{i}\right)$ defined in (3) where $\varepsilon$ is the sampling precision. Furthermore, the tensor metric is approximated by a constant tensor field equal to Ti over $B_{\varepsilon}\left(x_{i}\right)$. Under these assumptions, the discrete derivative free Eikonal equation reads

$$
\left\{\begin{array}{c}
\forall x_{i} \in \Omega, u_{i}=\min _{y \in \partial B_{\varepsilon}\left(x_{i}\right)} U(y)+d(y, x), \\
\forall x_{i} \in S, u_{i}=0,
\end{array}\right.
$$

Decomposing this minimization into each triangle (in 2D) of the neighborhood, and using the fact that $u(y)$ is affine on each triangle, one can re-write the discrete Eikonal equation as a fixed point

$$
u=\Gamma(u) \text { and } \forall x_{i} \in S, u_{i}=0
$$

where the operator $v=\Gamma(u) \in R^{n}$ is defined as:

where,

$$
v_{i}=\Gamma_{i}(u) \min _{t_{i, j, k} \in B_{\varepsilon}\left(x_{i}\right)} v_{i, j, k}
$$

$$
v_{i, j, k}=\min _{t \in B_{\varepsilon}[0,1]} t u_{j}+(1-t) u_{k}+\left\|t x_{j}+(1-t) x_{k}-x_{i}\right\|_{T_{i}^{-1}}
$$

We have written this equation for simplicity in the $2 D$ case, so that each point $y$ is a barycenter of two sampling points, but this extends to a manifold of arbitrary dimension $d$ by considering barycenters of $d$ points.

The discrete Eikonal equation is a non-linear fixed point problem. One can compute the solution to this problem using Jacobi iterations.

One can prove that the mapping $\Gamma$ is both monotones and non-expanding:

$$
\left\{\begin{array}{c}
u \leq \tilde{u} \rightarrow \Gamma(u) \leq \Gamma(\tilde{u}) \\
\|\Gamma(u)-\Gamma(\tilde{u})\| \leq\|u-\tilde{u}\|=\max \left|u_{i}-\tilde{u}_{i}\right|
\end{array}\right.
$$

These two properties enable the use of simple iterations that converge to the solution $u$ of the problem, one can apply the update operator $\Gamma$ to the whole set of grid points. Jabobi non-linear iterations initialize $u^{(0)}=0$ and then compute:

$$
u^{(k+1)}=\Gamma\left(u^{(k)}\right)
$$

The fixed point property is useful to monitor the convergence of iterative algorithms, since one stops iterations when one has computed some distance $u$ with

$$
\|\Gamma(u)-u\| \leq \mu \quad \text { where }\|u\|=\max _{i}\left|u_{i}\right|
$$


And where $\mu>0$ is some user-defined tolerance.

\subsection{Geodesic distance}

The geodesic distance between two points' $p_{0}$ and $p$ of $3 D$ face surface is the shortest path between the two points while remaining on the facial surface. In the context of calculating the geodesic distance $R$. Kimmel and J.A. Sethian [23] propose the method of Jacobi iteration as a solution of the Eikonal equation. The geodesic distance between two points on a surface is calculated as the length of the shortest path connecting the two points. Using the Jacobi iteration algorithm on the triangulated surface $3 D$ face, we can compute the geodesic distance between the reference point $p_{0}$ and the other point's $p$ constructing the facial surface. The geodesic distance $\delta_{p 0, p}$ between $p_{0}$ and $\mathrm{p}$ is approximated by the following expression:

$$
\delta_{p 0, p}=\min \gamma\left(\beta\left(p_{0}, p\right)\right)
$$

with: $\beta\left(p_{0}, p_{i}\right)$ is the path between $p_{0}$ and according to the surface $S$ of the $3 D$ face, and $\gamma\left(\beta\left(p_{0}, p\right)\right)$ is the path length.

The following Figure 4 shows the steps for determining the geodesic distance using a $3 D$ face image of SHREC2008 database.

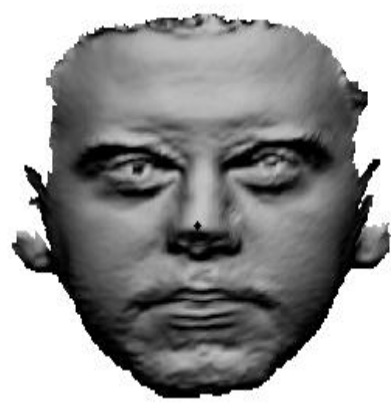

(a)

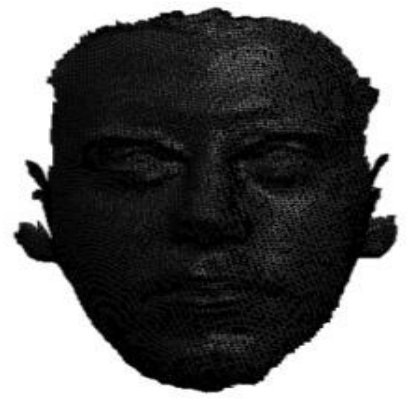

(b)

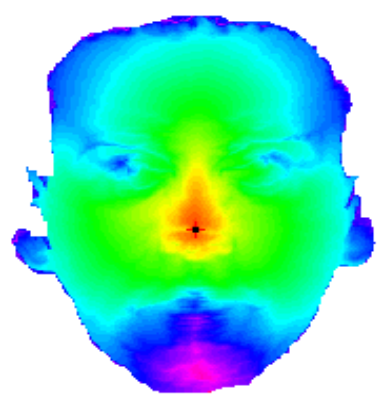

(c)

Figure 4. 3D face geodesic distance computes Steps: (a) Reference point detection; (b)

Discretization by triangular mesh; (c) Geodesic distance computing

Repeating this computation (geodesic distance $\delta_{p 0, p}$ ) between the reference point $p_{0}$ and each point $p$ of the surface $S$ of the $3 D$ face, then we compute a geodesic distance matrix $\Psi$ :

$$
[\Psi]=\delta \mathrm{ij}=\left[\begin{array}{ccc}
\delta_{11} & \cdots & \delta_{1 \mathrm{~m}} \\
\vdots & \ddots & \vdots \\
\delta_{\mathrm{n} 1} & \cdots & \delta_{\mathrm{nm}}
\end{array}\right]
$$

with, $\quad \delta_{i j}=\delta_{p 0, p}$

To realize our $3 D$ face recognition system, we use the Principal Component Analysis (PCA) and Linear Discriminant Analysis (LDA) for writing space of the geodesic distance matrix [ $\Psi$ ]. To realize our 3D face recognition system, we use three types of classification algorithms: the Neural Networks (NN), kNearest Neighbor (KNN) and Support Vector Machines (SVM).

\section{RESULTS AND ANALYSIS}

In this section we make a series of simulation to evaluate the effectiveness of the proposed approach. These results were performed based on SHREC 2008 database. This database contains total of 427 scans of 61 subjects (45 males and 16 females), for each of these 61 subjects 7 different scans, namely two "frontal", one "look-up", one "look-down", one "smile", one "laugh" and one "random expression" [26, 27]. 


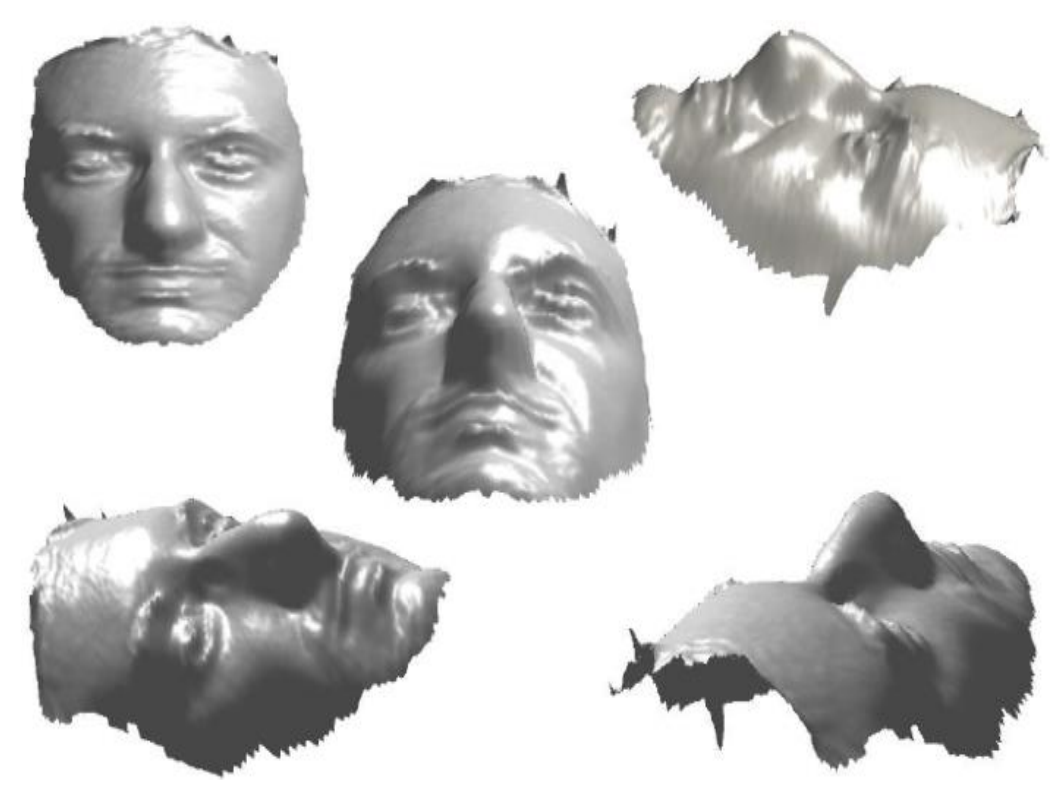

Figure 5. Example 3D face images of the database SHREC2008.

Figure 6 give the simulation results obtained by the proposed approach. This figure (6) shows the recognition rate found by the first method used in our 3D face recognition system such as Geodesic Distance using Jacobi Iteration (GD-JI) and Principal Component Analysis (PCA) for characters extraction, with three algorithms of classification: Neural Networks (NN), k-Nearest Neighbor (KNN) and Support Vector Machines (SVM). The results obtained by our 3D face recognition system shows that our methods are feasible, and present the best recognition rate $(98,6 \%)$ using SVM classifier for SHREC 2008 database images.

\section{GD-JI+PCA}

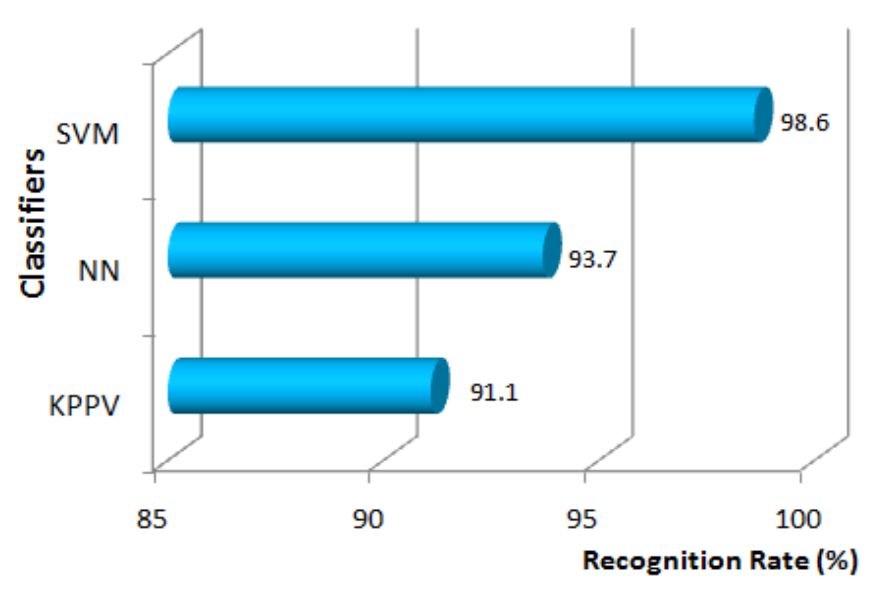

Figure 6. Recognition Rate for SHREC2008 images using GD-JI+PCA and three classification algorithms (NN, KNN and SVM)

In this seconde experiment, the features were extracted using Geodesic Distance computing with Jacobi Iterations (GD-JI) and Linear Discriminant Analysis (LDA). The simulation results of this method are presented in Figure 7. 


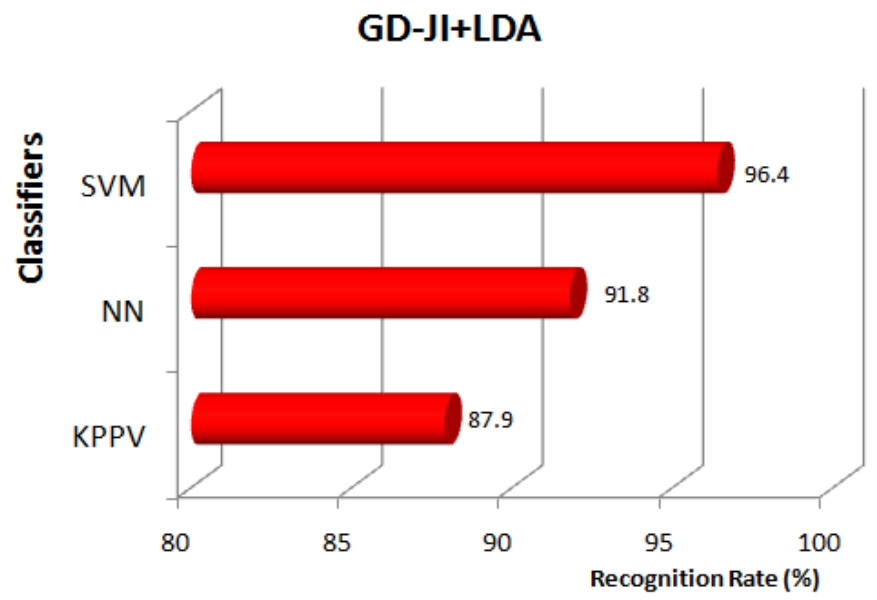

Figure 7. Recognition Rate for SHREC2008 images using GD-JI+LDA and three classification algorithms (NN, KNN and SVM)

Figure 7 shows the recognition rate found by the first method used in our 3D face recognition system such as Geodesic Distance using Jacobi Iteration and Linear Discriminant Analysis (GD-JI + LDA) for characters extraction, with three classification algorithms: Neural Networks (NN), k-Nearest Neighbor (KNN) and Support Vector Machines (SVM). The results obtained by our 3D face recognition system shows this method (GD+LDA) present the best recognition rate using SVM classification algorithm with $96,40 \%$ respectively for images of SHREC 2008 database.

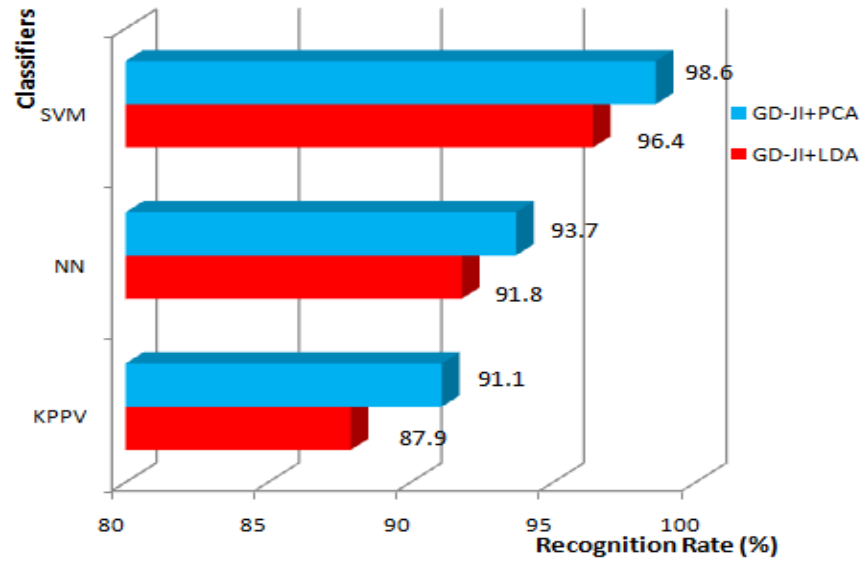

Figure 8. Comparative study of our methods GD-JI+PCA and GD-JI+LDA using three classification algorithms (NN, KNN and SVM)

In this paper, a number of face recognition algorithms have been described such as: GD-JI+PCA and GD-JI+LDA LDA using three classification algorithms (NN, KNN and SVM). These methods have been verified on the SHREC 2008 database, and the testing protocols used in the experiments are almost the same, so that a direct comparison of the results reported in these works is possible. In Figure 8 we give a comparison of these face recognition algorithms. Figure 8 shows a comparison recognition rate of these features extraction algorithms (GD-JI+PCA and GD-JI+LDA LDA) using SHREC 2008 database images. This comparative study gives the best recognition rate $(98.6 \%)$ was presented for GD-JI+PCA using SVM classifier, then this method was also better than other tree approaches.

\section{CONCLUSION}


In this work, we have presented a 3D face recognition system based on the computation of the geodesic distance between the reference point and the other points in the 3D face surface using Jacobi iterations. For Geodesic distances space matrices reduction, we used Principal Component Analysis (PCA) or Linear Discriminant Analysis (LDA) algorithms. For the classifying steps we have implemented algorithms as NN, KNN and SVM. Simulation results show us a better recognition rate (98.6\%) for was presented for GDJI+PCA using SVM classifier, then this method was also better than other tree approaches. In future work, we will take a 3D face recognition system by analyzing the iso-geodesic curves using Riemannian geometry and compare these results with those obtained by this method.

\section{REFERENCES}

[1] C. Chua; F. Han; Y.K. Ho, "3D human face recognition using point signature," IEEE International Conference on Automatic Face and Gesture Recognition, pp.233-238, 2000.

[2] B. Achermann; H. Bunke , "Classifying range images of human faces with Hausdorff distance," in: 15 th International Conference on Pattern Recognition, pp. 809-813, September 2000.

[3] A.B. Moreno; A' ngel Sa'nchez; J.F. Ve'lez; F.J. D1'az , "Face recognition using 3D surface-extracted descriptors, " in: Irish Machine Vision and Image Processing Conference (IMVIP 2003), September 2003.

[4] Y. Lee; K. Park; J. Shim; T. Yi , "3D face recognition using statistical multiple features for the local depth information," in: 16th International Conference on Vision Interface, June 2003.

[5] G. Medioni; R. Waupotitsch , "Face recognition and modeling in 3D," in: IEEE International Workshop on Analysis and Modeling of Faces and Gestures (AMFG 2003), pp. 232-233, October 2003.

[6] C. Xu; Y. Wang; T. Tan; L. Quan , Automatic 3D face recognition combining global geometric features with local shape variation information," in: Sixth International Conference on Automated Face and Gesture Recognition, pp. 308-313, May 2004.

[7] X. Lu; D. Colbry; A.K. Jain, "Matching 2.5D scans for face recognition,” in: International Conference on Pattern Recognition (ICPR 2004), pp. 362-366, 2004.

[8] G. Pan; S. Han; Z. Wu; Y. Wang, "3D face recognition using mapped depth images," in: IEEE Workshop on Face Recognition Grand Challenge Experiments, June 2005.

[9] Y. Lee; H. Song; U. Yang; H. Shin; K. Sohn , "Local feature based 3D face recognition," in: International Conference on Audio- and Video based Biometric Person Authentication (AVBPA 2005), LNCS, vol. 3546, pp. 909-918, July 2005.

[10] Y. Wang; G. Pan; Z. Wu; Y. Wang , "Exploring facial expression effects in 3D face recognition using partial IC," in P. Narayanan (ed.), Computer Vision "U ACCV 2006, (3851) 581-590 of Lecture Notes in Computer Science, Springer Berlin/Heidelberg, 2006.

[11] C. Samir; A. Srivastava; M. Daoudi , "Three- Dimensional Face Recognition Using Shapes of Facial Curves," In IEEE Transactions On Pattern Analysis And Machine Intelligence, pp..1858-1863, 2006.

[12] S. Feng; H. Krim; I.A Kogan , "3D face recognition using euclidean integral invariants signature.” In SSP '07: IEEE/SP 14th Workshop on Statistical Signal Processing, 156-160, Madison, WI, USA, 2007.

[13] S. Jahanbin; H. Choi; Y. Liu, A. C. Bovik, "Three dimensional face recognition using iso-geodesic and iso-depth curves," In BTAS '08: Proceedings of the IEEE Second International Conference on Biometrics Theory, Applications and Systems, Arlington, Virginia, USA, 2008.

[14] X. Han; H. Ugail; I. Palmer " "Method of Characterising 3D Faces Using Gaussian Curvature," In IEEE, 2009.

[15] H. drira; B. Ben Amor; A. Srivastava; M. Daoudi , "A Riemannian Analysis of 3D Nose Shapes For Partial Human Biometrics," In 12th International Conference on Computer Vision (ICCV), IEEE, 2009.

[16] A. Maalej; B. Ben Amor; M. Daoudi; A. Srivastava; S. Berretti , "Local 3D Shape Analysis for Facial Expression Recognition," In IEEE, International Conference on Pattern Recognition, 2010.

[17] Bronstein A. M;, Bronstein M. M.;, Kimmel R. , "Threedimensional face recognition,” International Journal of Computer Vision, vol.64, no.1, pp.5-30, 2005.

[18] C. Xu; Y. Wang; T. Tan; L. Quan, "Robust nose detection in 3D facial data using local characteristics," Image Processing, ICIP '04. 2004 International Conference on, vol.3, pp. 1995-1998, 2004.

[19] L.H. Anuar; S. Mashohor; M. Mokhtar; W.A. Wan Adnan, "Nose Tip Region Detection in 3D Facial Model across Large Pose Variation and Facial Expression," IJCSI International Journal of Computer Science Issues, vol.7, Issue 4, no.4, July 2010.

[20] S. Jahanbin; H. Choi; Y. Liu; A. C. Bovik, "Three Dimensional Face Recognition Using Iso-Geodesic and IsoDepth Curves," Biometrics: Theory, Applications and Systems, 2008. BTAS 2008, 2nd IEEE International Conference on, 2008. SSIAI 2008 , pp. 1 - 6, Sept. 29 2008-Oct. 12008.

[21] A. Salima; S. Salim; T. A. Abdelmalik , "A Survey of Approaches for Curve Based Facial Surface Representations For Three-Dimensional Face Recognition."

[22] R. Kimmel; J. A. Sethian, “Computing geodesic on manifolds, ” in Proc. US National Academy of Science, vol. 95, pp. 8431-8435, 1998.

[23] Xavier Desquesnes; Abderrahim Elmoataz; Olivier Lézoray," Eikonal equation adaptation on weighted graphs: fast geometric diffusion process for local and non -local image and data processing, " Journal of Mathematical Imaging and Vision 46, vol.2, pp. 238-257, 2014. 
[24] Carlini, E.; Falcone; M., Forcadel; N., Monneau , "R.: Convergence of a generalized fast-marching method for an eikonal equation with a velocity-changing sign," SIAM J. Numer. Anal. 46, pp.2920- 2952, 2008.

[25] Frank B. ter Haar; Mohamed Daoudi; Remco C , Veltkamp: SHape REtrieval Contest 2008: 3D Face Scans. "Shape Modeling and Applications, 2008. SMI 2008. IEEE International Conference on, 4-6 June 2008.

[26] Brian Amberg; Reinhard Knothe; Thomas Vetter, "SHREC'08 Entry: Shape Based Face Recognition with a Morphable Model,” Shape Modeling and Applications, 2008. SMI 2008. IEEE International Conference on, pp.4-6 June 2008.

[27] C. Samir; M. Daoudi; A. Srivastava , “A Framework of Calculus on Facial Surfaces," In 14th International Conference of Image Analysis and Processing - Workshops , ICIAPW , 2007. 